\title{
Formation of Iron Oxide Nanorods using Electron Beam-Induced Deposition
}

\author{
M. Shimojo,***, M. Takeguchi ${ }^{*}$, W. Lu ${ }^{*}$ and K. Furuya ${ }^{*}$ \\ *High Voltage Electron Microscopy Station, National Institute for Materials Science, \\ 3-13 Sakura, Tsukuba, 305-0003, Japan \\ ** Advanced Science Research Laboratory, Saitama Institute of Technology, \\ 1690 Fusaiji, Fukaya, 369-0293, Japan
}

Electron beam-induced deposition (EBID) is a promising technique to fabricate nanometer-sized deposits such as dots and wires without using any masks. In this technique, organometallic precursors are decomposed by focused electron beams. The shape and position of the nano-deposits can be controlled by controlling the beam position. The resolution of EBID is now reaching down to sub-nanometers [1]. However, the deposits fabricated by EBID generally contain a large amount of carbon that is originated from the organometallic precursor and from the residual hydrocarbon gases inside the chamber. Transmission electron microscopy study revealed that the deposits obtained by EBID consisted of amorphous carbon or nanometer-sized crystals embedded in an amorphous carbon matrix [2].

As the composition and crystallographic structure of nanomaterials greatly affect their physical properties [3], it is necessary to control the composition and crystal structure of the nano-deposits and to expand the range of materials that can be produced by EBID, in order to apply an EBID technique to the production of nanometer-sized devices. Some researchers have tried to improve the purity. Folch [4] reported the reduction of carbon to $50 \%$ by adding oxygen to the precursor. However, even for the oxygen addition to organometallic precursors, the deposition of high purity metals or oxides was not reported. In this work, the direct formation of oxides without carbon by EBID at room temperature is studied.

A field emission gun scanning electron microscope (SEM, JEOL JSM-7800UHV) having a gas introduction system was used for EBID experiments. The base pressure of the specimen chamber was about $2 \times 10^{-6} \mathrm{~Pa}$. The accelerating voltage and beam current used for deposition were $30 \mathrm{kV}$ and $8 \times 10^{-10} \mathrm{~A}$, respectively. The nominal probe diameter was $4 \mathrm{~nm}$. A schematic illustration of the deposition system is shown in Fig. 1. Two cylinders, including iron pentacarbonyl and water, were placed simultaneously. Vapors of these two were mixed and introduced into the chamber through a nozzle. The electron beam position was controlled using a computer. All the deposition was carried out at room temperature. After deposition, the deposits were observed using a transmission electron microscope (TEM, JEOL JEM-3000F) with a Gatan imaging filter.

Figure 2 shows a scanning electron micrograph of a nanorod formed using an iron pentacarbonyl precursor with water vapor. The nanorod was about $300 \mathrm{~nm}$ in length and $30 \mathrm{~nm}$ in width. Figure 3 shows electron energy loss spectra of the nanorod fabricated with and without mixing water vapor. Iron, oxygen and carbon edges are observed in 
the spectrum of the nanorod fabricated without water vapor, but no carbon edges are seen in that with water vapor. This indicates that carbon contamination was successfully removed by mixing water vapor to the precursor vapor, and that iron oxide nanorod was considered to be formed by EBID at room temperature.

\section{References}

[1] W. F. van Dorp, et al., Nano Lett. 5 (2005) 1303.

[2] M. Han, et al., Philos. Mag. 21 (2004) 1281.

[3] Y. Tsukatani, et al., Jpn. J. Appl. Phys. 44 (2005) 5683.

[4] A. Folch, et al., Appl. Phys. Lett. 66 (1995) 2080.

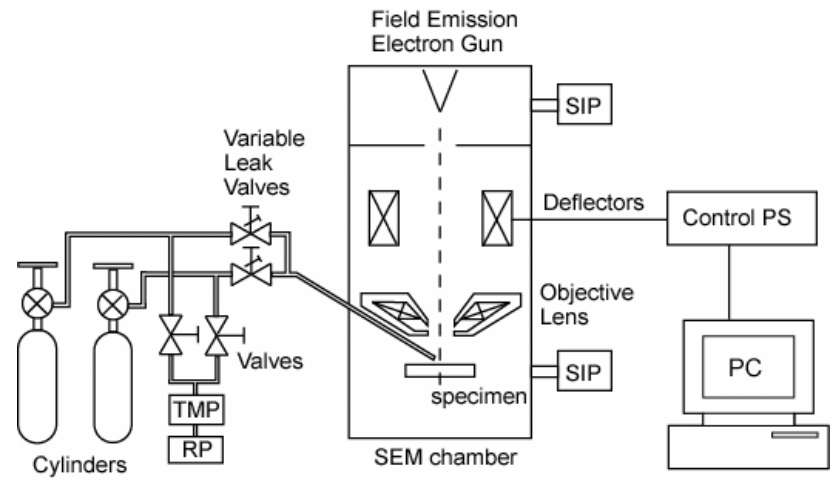

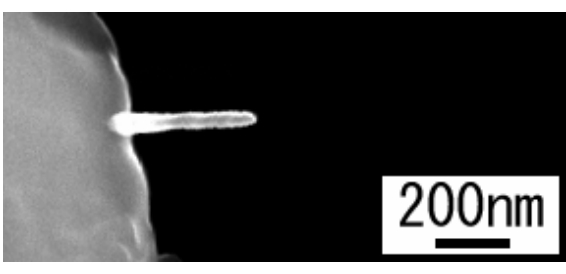

Fig. 2 SEM image of a nanorod formed

Fig. 1 Schematic illustration of the deposition system
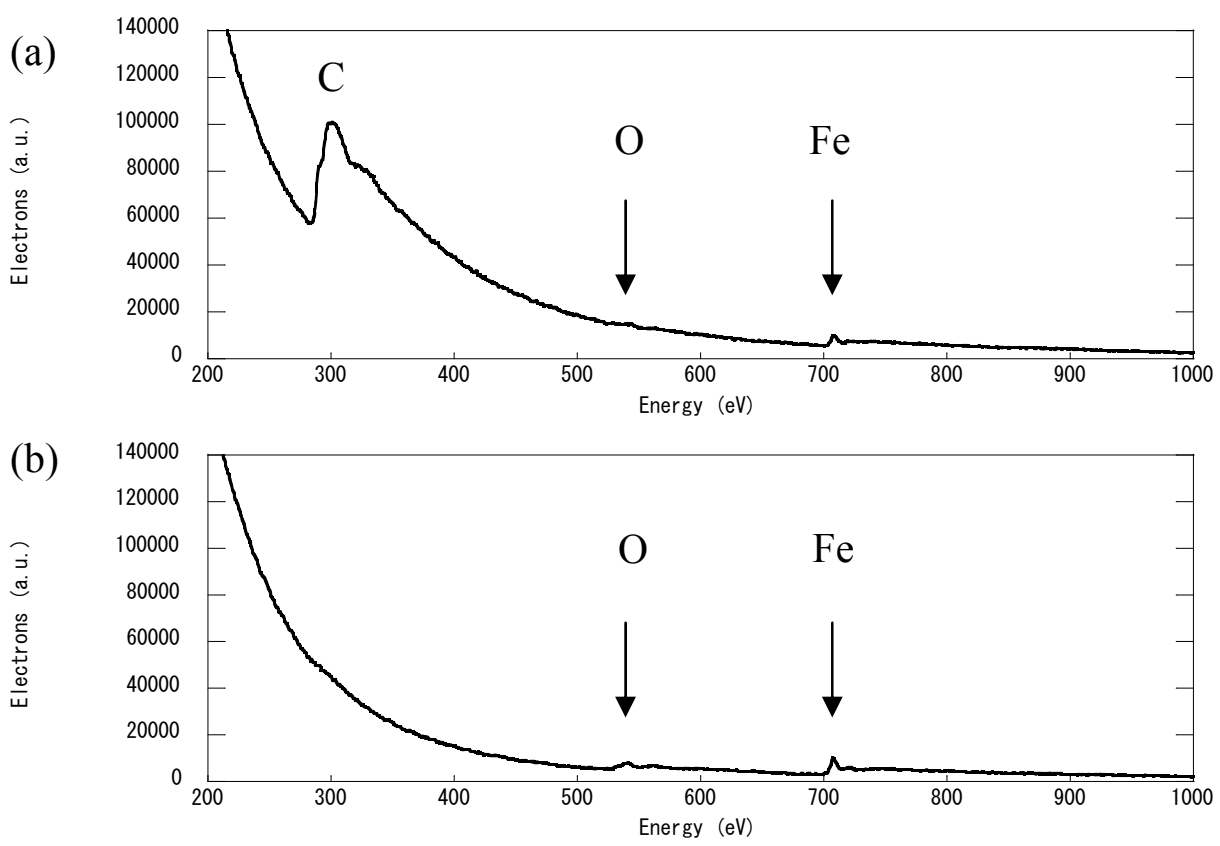

Fig. 3 EELS spectra of nanorods formed using $\mathrm{Fe}(\mathrm{CO})_{5}$ only (a) and $\mathrm{Fe}(\mathrm{CO})_{5}$ with water (b) 